\title{
Energy spectrum for two-dimensional potentials in very high magnetic fields
}

\author{
Z. Gedik and M. Bayindir \\ Department of Physics, Bilkent University, Bilkent 06533 Ankara, Turkey
}

(Received 10 March 1997; revised manuscript received 29 May 1997)

\begin{abstract}
A method, analogous to supersymmetry transformation in quantum mechanics, is developed for a particle in the lowest Landau level moving in an arbitrary potential. The method is applied to two-dimensional potentials formed by Dirac $\delta$ scattering centers. In the periodic case, the problem is solved exactly for rational values of the magnetic flux (in units of flux quantum) per unit cell. The spectrum is found to be self-similar, resembling the Hofstadter butterfly [Phys. Rev. B 14, 2239 (1976)]. [S0163-1829(97)06436-9]
\end{abstract}

In recent years, the energy spectrum of two-dimensional electron systems have attracted great interest, because of the relevance of the problem to the magnetotransport properties ${ }^{1}$ and in particular to the quantum Hall effect. ${ }^{2}$ It is believed that the physics of the integer quantum Hall effect is governed by the interaction of electrons with a disordered potential, which leads to a localization of the eigenfunctions. Observed conductance steps can be explained by a sequence of localization-delocalization transitions. Quantization of the Hall conductance due to periodic potentials has also been studied. ${ }^{3,4}$ In this case, the presence of steps is explained by the gaps in the energy spectrum.

There are two opposite approaches to the problem of an electron moving in a periodic potential: the tight-binding and the nearly-free-electron methods. In the first approach, the magnetic field is introduced via Peierls substitution, where the matrix elements are multiplied by $\exp \left[(i q / \hbar c) \int \mathbf{A} \cdot d \mathbf{l}\right] .^{5}$ On the other hand, in the case of nearly free electrons, the Landau-level structure is essential, and the lattice potential is introduced via intra- and inter-Landau-level scattering matrix elements. The duality between the position and the momentum in quantum mechanics leads to similarities between the two methods. In the presence of a magnetic field, the secular equations for the two limits, the tight-binding and the nearlyfree-electron approach, with certain approximations, are identical. ${ }^{6}$ The characteristic feature of the problem is that the secular determinant for the limit of infinite crystals can be reduced to a finite determinant, when the magnetic flux per unit cell is a rational number in units of the flux quantum. The tight-binding case was studied by Azbel' 7 and Hofstadter, ${ }^{8}$ who showed that the system has a complicated self-similar spectrum.

In this work, an approach is developed for a particle in the lowest Landau level moving in an arbitrary potential. The method is used to obtain the energy spectrum in the presence of Dirac $\delta$ potentials. The difficulty with the Dirac $\delta$ potential is that, even if the inter-Landau-level couplings are small and can be neglected, there is a strong intra-Landau-level mixing. The formalism developed in this study leads to an eigenvalue problem where the coupling between distant sites become negligibly small. Moreover, since the problem is formulated in real space, the distribution of Dirac $\delta$ potentials can be arbitrary. In spite of the sharpness of the potential, the assumption that a particle is confined into a single Landau level is consistent with the result that shifts in energy eigen- values are very small in comparison to inter-Landau-level separation. The method can be used for any potential, as far as the particle is confined into the lowest Landau level. After presenting the method, the case of Dirac $\delta$ scattering centers will be solved for periodic distribution.

The Hamiltonian for a particle of mass $m$ and charge $q$, moving in two dimensions in the presence of magnetic field $\mathbf{B}=\nabla \times \mathbf{A}$, perpendicular to the plane and potential $V$, is given by $H=H_{0}+V$, where

$$
H_{0}=\frac{1}{2 m}\left(\mathbf{p}-\frac{q}{c} \mathbf{A}\right)^{2} .
$$

Using the symmetric gauge $\mathbf{A}=\frac{1}{2} \mathbf{B} \times \mathbf{r}$ and complex coordinates $z=X+i Y=\sqrt{q B / 2 \hbar c}(x+i y)$, where $\mathbf{r}=(x, y)$, the unperturbed Hamiltonian can be written as $H_{0}=a^{\dagger} a$, where $a^{\dagger}=-\partial / \partial z+z^{*} / 2$. Since $\left[a, a^{\dagger}\right]=1$, the energy eigenvalues are given by $E_{n}=\hbar \omega(n+1 / 2)$, where $\omega=q B / 2 m c$ ( $q$ is assumed to be positive) and $n=0,1,2, \ldots$ The ground-state wave functions are of the form $f(z) e^{-|z|^{2} / 2}$. Here $f(z)$ is any analytic function of $z$. A basis for the infinitely degenerate ground state is formed by

$$
\phi_{m}\left(z, z^{*}\right)=\frac{z^{m}}{\sqrt{\pi m !}} e^{-|z|^{2} / 2},
$$

with $m=0,1,2, \ldots$ When the magnetic field is very high the particle is confined into the lowest Landau level. This is a good approximation as long as the potential is small in comparison to Landau-level splitting $\hbar \omega$. The problem is reduced to the diagonalization of the matrix $V$, whose entries are given by

$$
\left\langle m|V| m^{\prime}\right\rangle=\iint \phi_{m}^{*}\left(z, z^{*}\right) V\left(z, z^{*}\right) \phi_{m}\left(z, z^{*}\right) d^{2} z,
$$

where $d^{2} z=d x d y$. Let $A$ and $B$ be defined by

$$
\begin{aligned}
& A_{m, z z^{*}}=\phi_{m}^{*}\left(z, z^{*}\right) \sqrt{V\left(z, z^{*}\right)}, \\
& B_{z z^{*}, m}=\sqrt{V\left(z, z^{*}\right)} \phi_{m}\left(z, z^{*}\right) .
\end{aligned}
$$

Therefore $V=A B$. For positive $V\left(z, z^{*}\right), A=B^{\dagger}$. Now consider the operator $\widetilde{V}=B A$. Any eigenvalue $E$ associated with the eigenket $|\widetilde{\psi}\rangle$ of the operator $\widetilde{V}=B A$ is also an eigenvalue 
of the operator $V=A B$, except when $A|\widetilde{\psi}\rangle=0$. The corresponding ket is given by $|\psi\rangle=E^{-1 / 2} A|\widetilde{\psi}\rangle$. Similarly, any eigenvalue $E$ associated with the eigenket $|\psi\rangle$ of the operator $V=A B$ is also an eigenvalue of the operator $\widetilde{V}=B A$, except when $B|\psi\rangle=0$, and the corresponding ket is given by $|\widetilde{\psi}\rangle=E^{-1 / 2} B|\psi\rangle$. Therefore it is enough to diagonalize $\widetilde{V}$, instead of $V$, to find the energy eigenvalues, except for the ground state. This method is analogous to supersymmetric quantum mechanics pioneered by Witten. ${ }^{9}$ Substitution of $A$ and $B$ yields

$$
\begin{aligned}
\left\langle z z^{*}|\widetilde{V}| z^{\prime} z^{\prime *}\right\rangle= & \left(\frac{V\left(z, z^{*}\right)}{\pi}\right)^{1 / 2} e^{z z^{\prime *}-|z|^{2} / 2-\left|z^{\prime}\right|^{2 / 2}} \\
& \times\left(\frac{V\left(z^{\prime}, z^{\prime *}\right)}{\pi}\right)^{1 / 2} .
\end{aligned}
$$

When $V\left(z, z^{*}\right)$ is negative, $\widetilde{V}=B A$ is not Hermitian, but the eigenvalues are still real. The method can be applied to any problem in the lowest Landau level, but its advantages will be demonstrated for the potential

$$
V(\mathbf{r})=V_{0} \sum_{i} \delta\left(\mathbf{r}-\mathbf{r}_{i}\right) .
$$

In this case, $z$ and $z^{\prime}$ become discrete variables, and the matrix to be diagonalized is

$$
\langle i|\widetilde{V}| j\rangle=\frac{V_{0}}{\pi} e^{z_{i} z_{j}^{*}-\left|z_{i}\right|^{2} / 2-\left|z_{j}\right|^{2} / 2} .
$$

It is interesting to note that the matrix elements are very similar to those of the tight-binding problem. Phase is nothing but the usual Peierls factor. $\langle i|\widetilde{V}| j\rangle$ and $\left\langle m|V| m^{\prime}\right\rangle$ have the same spectrum except for the ground state. Let $N_{i}$ be the number of impurities and $N$ be the number of states, both of which go to infinity, so that $N_{i} / N \rightarrow \rho$. Hence $\widetilde{V}$ and $V$ have $N_{i}$ and $N$ eigenvalues, respectively. The larger one has $\left|N_{i}-N\right|$ zero eigenvalues. If $N_{i}<N, N-N_{i}$ eigenvalues of $V$ will be zero. $\widetilde{V}$ filters the nonzero eigenvalues of $V$. However, if $\widetilde{V}$ itself gives zero eigenvalues, the situation must be examined carefully to distinguish the zero eigenvalues from the extra zeros. Once $\widetilde{V}$ is diagonalized, the eigenfunctions of $V$ can be constructed by acting $A$ on the eigenvectors of $\widetilde{V}$. The impurities can be distributed in an arbitrary way.

The problem of an electron in a magnetic field interacting with point impurities has been discussed extensively in the literature. ${ }^{10-15}$ It has been shown that the zeros of the wave function can be adjusted to coincide with the locations of the scatterers if the concentration of the scatterers is low enough. This corresponds to the $N_{i}<N$ case discussed above. The presence of $N-N_{i}$ energy eigenvalues unaffected by the impurities is a direct consequence of the dimensionality of the matrix to be diagonalized. Furthermore, the method gives the full energy spectrum for any distribution of impurities. It must be noted that Dirac $\delta$ potentials have some peculiarities in two dimensions. They do not cause scattering, irrespective of their strength. However, in the presence of a magnetic field energy and length scales are introduced, and they lead to nontrivial spectra.
For regularly distributed impurities the problem can be handled by introducing periodic solutions. For simplicity the square lattice case will be investigated. Generalization to an arbitrary lattice is straightforward. Now, the impurity positions are given by $\mathbf{r}_{m n}=d(m, n)$, where $m$ and $n$ are integers and $d$ is the lattice constant. The matrix elements become

$$
\begin{aligned}
\left\langle m n|\widetilde{V}| m^{\prime} n^{\prime}\right\rangle= & \frac{V_{0}}{\pi} \exp \left[-\pi \frac{\phi}{\phi_{0}}\left(\frac{\left(m-m^{\prime}\right)^{2}+\left(n-n^{\prime}\right)^{2}}{2}\right.\right. \\
& \left.\left.+i\left(m n^{\prime}-n m^{\prime}\right)\right)\right],
\end{aligned}
$$

where $\phi$ is the magnetic flux passing through a unit cell (or flux per impurity), and $\phi_{0}$ is the flux quantum (note that the ratio of the area of the system to the number of states is $\pi$ ). When $\phi / \phi_{0}$ is a rational number the system becomes periodic. Let $\phi / \phi_{0}=\alpha=p / q$, where $p$ and $q$ are relatively prime integers then $\left\langle m n|\widetilde{V}| m^{\prime} n^{\prime}\right\rangle$ is left invariant under $2 q$ translations along lattice axes. If $p$ is even, the matrix elements become periodic with period $q$ instead of $2 q$. In any case, as long as $\alpha$ is rational, the eigenvectors $|n \mathbf{k}\rangle$ have the property that

$$
\langle\mathbf{R} \tau \mid n \mathbf{k}\rangle=\langle 0 \tau \mid n \mathbf{k}\rangle e^{i \mathbf{k} \cdot \mathbf{R}},
$$

where $\mathbf{R}$ fixes the $2 q \times 2 q$ (or $q \times q$ ) unit cell and $\tau$ denotes the position of the impurity in the cell. Here $n$ is an integer (band index) and $\mathbf{k}$ is a two-dimensional vector. Hence the problem is reduced to determination of the coefficients $\langle 0 \tau \mid n \mathbf{k}\rangle$, which satisfy

$$
\sum_{\tau} \widetilde{V}_{\tau \tau^{\prime}}(\mathbf{k})\left\langle 0 \tau^{\prime} \mid n \mathbf{k}\right\rangle=E_{n \mathbf{k}}\langle 0 \tau \mid n \mathbf{k}\rangle
$$

where

$$
\widetilde{V}_{\tau \tau^{\prime}}(\mathbf{k})=\sum_{\mathbf{R}}\left\langle\mathbf{R} \tau|\widetilde{V}| 0 \tau^{\prime}\right\rangle e^{i \mathbf{k} \cdot \mathbf{R}} .
$$

Given the flux ratio $\alpha$, the energy eigenvalues $E_{n \mathbf{k}}$ can be found by diagonalizing $4 q^{2} \times 4 q^{2}$ matrix $\widetilde{V}_{\tau \tau^{\prime}}(\mathbf{k})$. However, this does not imply that there will be $4 q^{2}$ energy bands. If the problem is formulated in the Landau gauge, the additional symmetry can be seen easily. It turns out that unit cell has dimensions $1 \times q$, and hence there will be $q$ bands. The symmetric gauge leads to a simple formulation of the problem but in the meantime some symmetries are obscured.

In Fig. 1 energy bands for $\alpha=\frac{4}{3}$ and $\alpha=\frac{10}{7}$ are plotted. There are three and seven energy bands, respectively. Using the relation $|\psi\rangle=E^{-1 / 2} A|\widetilde{\psi}\rangle$, it is possible to find the corresponding eigenfunction $\psi(\mathbf{r})$ of the original problem. Since $\psi(\mathbf{r})$ belongs to the lowest Landau level it must be in the form of $f(z) e^{-|z|^{2 / 2}}$, where $f(z)$ is any analytic function. Analytic functions are determined by their zeros. Considering the translational invariance of the system one expects to have regularly distributed zeros. Figure 2 shows the contour plots of $|\psi(\mathbf{r})|$ for $\alpha=2$. This value of the flux ratio is very special in that all of the matrix elements $\left\langle m n|\widetilde{V}| m^{\prime} n^{\prime}\right\rangle$ become real, and the problem reduces to a tight-binding sys- 


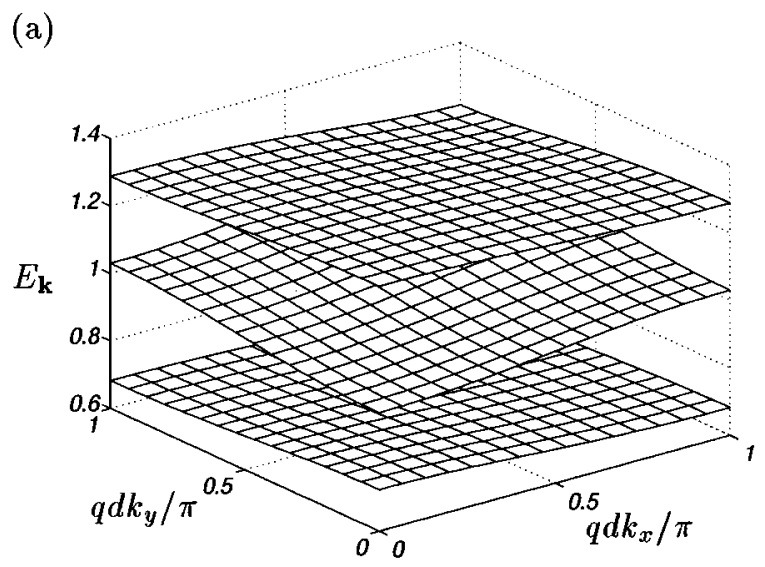

(b)

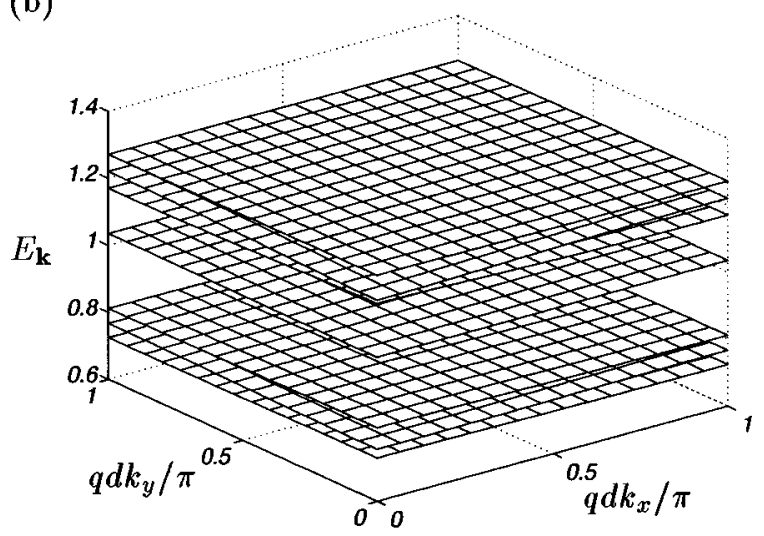

FIG. 1. Energy bands over one-quarter of the magnetic Brillouin zone for (a) $\alpha=\frac{4}{3}$ and (b) $\alpha=\frac{10}{7}$. The energy eigenvalues $E_{\mathbf{k}}$ are plotted in units of $V_{0} / \pi$.
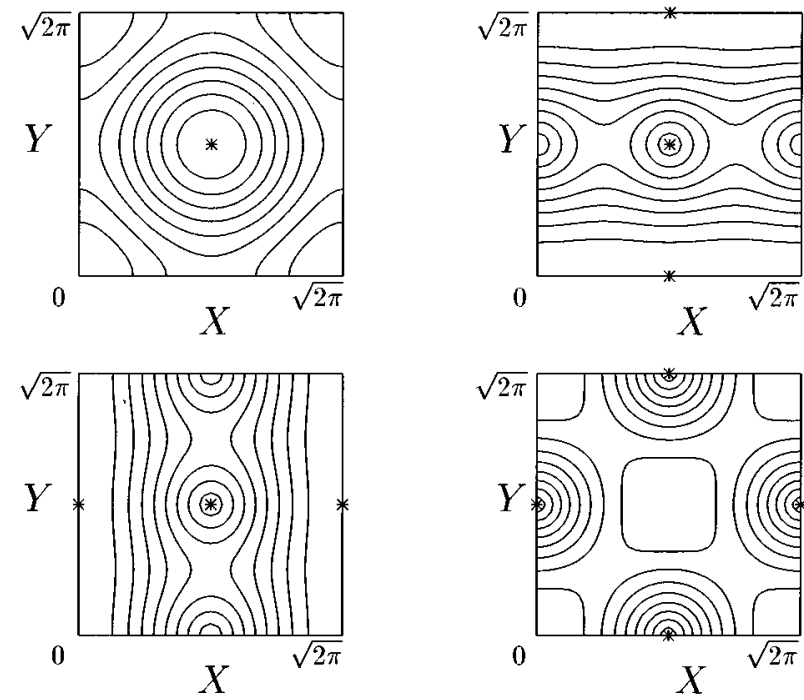

FIG. 2. Contour plots of $\left|\psi_{\mathbf{k}}(\mathbf{r})\right|$ for $\alpha=2$. Zeros are marked with *. (a) $k_{x}=k_{y}=0$, (b) $k_{x}=\sqrt{\pi / 2}$ and $k_{y}=0$, (c) $k_{x}=0$ and $k_{y}=\sqrt{\pi / 2}$, and (d) $k_{x}=k_{y}=\sqrt{\pi / 2}$.

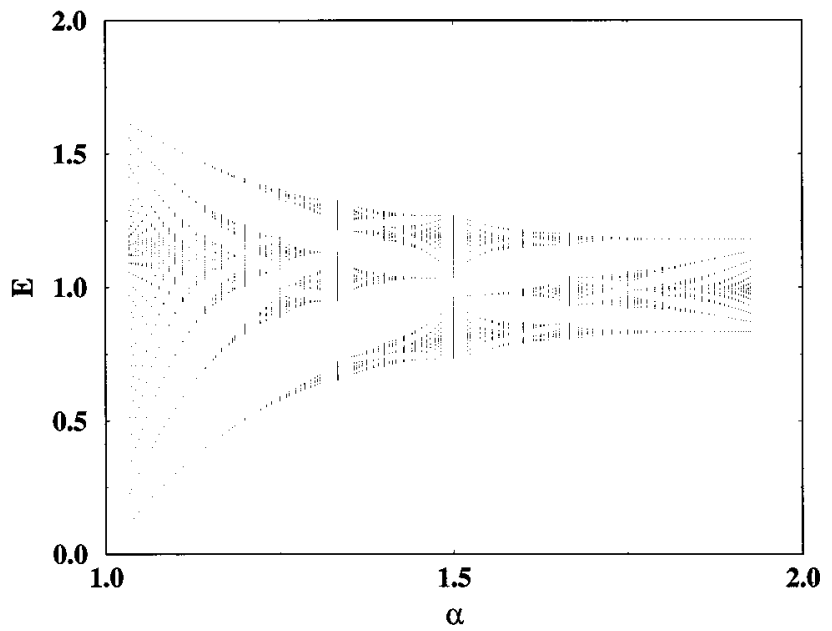

FIG. 3. Energy eigenvalues vs magnetic flux (in units of flux quantum) per unit cell for square lattice.

tem. For this particular case, energy eigenvalues $E_{\mathbf{k}}$ and eigenfunctions $\psi_{\mathbf{k}}$ can be found explicitly in terms of the theta function

$$
\theta_{3}(z \mid \tau)=\sum_{n=-\infty}^{\infty} \exp \left(\pi i \tau n^{2}\right) \exp (2 n \pi i z)
$$

which vanishes at $z=(l+1 / 2)+(m+1 / 2) \tau$, where $l$ and $m$ are any integers. It can be shown that

$$
E_{\mathbf{k}}=\frac{V_{0}}{\pi} \theta_{3}\left(\frac{k_{x}}{\sqrt{2 \pi}} \mid 2 i\right) \theta_{3}\left(\frac{k_{y}}{\sqrt{2 \pi}} \mid 2 i\right)
$$

and

$$
\psi_{\mathbf{k}}\left(z, z^{*}\right)=c e^{-|z|^{2} / 2} \theta_{3}\left(\frac{z+i k_{x}}{i \sqrt{2 \pi}} \mid i\right) \theta_{3}\left(\frac{z-k_{y}}{\sqrt{2 \pi}} \mid i\right)
$$

where $d$ is the lattice constant and $c$ is chosen to normalize $\psi_{\mathbf{k}}\left(z, z^{*}\right)$ properly. $\psi_{\mathbf{k}}\left(z, z^{*}\right)$ has regularly distributed zeros. For $\mathbf{k}=0$, the zeros of the two $\theta_{3}$ functions coincide, and they become double zeros. For $\mathbf{k} \neq 0$, they shift by an amount $-i k_{x}$ and $k_{y}$, respectively.

Figure 3 shows energy (measured in units of $V_{0} / \pi$ ) versus the flux ratio for the square lattice. Here the labels $n$ and $\mathbf{k}$ have been suppressed, i.e., $E_{n \mathbf{k}}$ values have been projected on to the energy axis. The energy spectrum is found to be self-similar, i.e., the same pattern is repeated with a different size and in a slightly distorted shape. With increasing flux ratio, the spectrum is squeezed since the amplitude of the matrix elements decreases with distance in a Gaussian way.

In conclusion, a method for the evaluation of the energy spectrum of a particle in the lowest Landau level, moving in an arbitrary potential, has been developed. The problem of Dirac $\delta$ scattering centers has been solved for demonstration. The periodic case was found to have a self-similar spectrum. The method is very general, since it can be applied not only to any distribution of impurities but any potential, as far as the particle is confined into the lowest Landau level. For the random distribution of scatterers, ${ }^{16}$ the calculated density of states is in perfect agreement with the analytic result of 
Brezin, Gross, and Itzykson. ${ }^{17}$ For a continuous potential, the matrix elements are labeled by continuous indices, and hence the proposed approach may not be as practical as it is in the Dirac $\delta$ type potential case.
This work was partially supported by the Scientific and Technical Research Council of Turkey under Grant No. TBAG-AY/123. One of the authors (Z.G.) is grateful to O. Tekman for helpful conversations.
${ }^{1}$ D. Pfannkuche and R. R. Gerhardts, Phys. Rev. B 46, 12606 (1992).

${ }^{2}$ T. Chakraborty and P. Pietiläinen, The Quantum Hall Effects: Fractional and Integral (Springer-Verlag, Berlin, 1995).

${ }^{3}$ P. Streda, J. Phys. C 15, L1299 (1982).

${ }^{4}$ D. J. Thouless, M. Kohmoto, M. P. Nightingale, and M. den Nijs, Phys. Rev. Lett. 49, 405 (1982).

${ }^{5}$ R. E. Peierls, Z. Phys. 80, 763 (1933).

${ }^{6}$ D. Langbein, Phys. Rev. 180, 633 (1969).

${ }^{7}$ M. Ya. Azbel', Zh. Eksp. Teor. Fiz. 46, 435 (1964) [Sov. Phys. JETP 19, 292 (1964)].

${ }^{8}$ D. Hofstadter, Phys. Rev. B 14, 2239 (1976).
${ }^{9}$ E. Witten, Nucl. Phys. B 188, 513 (1981).

${ }^{10}$ R. E. Prange, Phys. Rev. B 23, 4802 (1981).

${ }^{11}$ T. Ando, J. Phys. Soc. Jpn. 52, 1740 (1983).

${ }^{12}$ Y. Avishai and R. M. Redheffer, Phys. Rev. B 47, 2089 (1993).

${ }^{13}$ D. Huang and G. Gumbs, Phys. Rev. B 47, 9597 (1993).

${ }^{14}$ R. Brako and Z. Crljen, Phys. Rev. B 47, 13568 (1993).

${ }^{15}$ K. Ishikawa, N. Maeda, and K. Tadaki, Phys. Rev. B 51, 5048 (1995).

${ }^{16}$ Z. Gedik and M. Bayindir (unpublished).

${ }^{17}$ E. Brézin, D. J. Gross, and C. Itzykson, Nucl. Phys. B 235, 24 (1984). 